\title{
Adquisición de Competencias Transversales: Comparación entre los Grados en Estadística y Empresa, Administración y Dirección de Empresas y Finanzas y Contabilidad de la Universidad de Jaén
}

M. Virtudes Alba-Fernández ${ }^{(1)}$, Francisca Jiménez-Jiménez ${ }^{(2)}$, M. Paz Horno-Bueno $^{(3)}$, Rocío Martínez-Jiménez ${ }^{(4)}$, Antonia Oya-Lechuga ${ }^{(1)}$

(1) Departamento de Estadística e Investigación Operativa, Universidad de Jaén, Campus Las Lagunillas, Jaén, e-mail: mvalba@ujaen.es, aoya@ujaen.es

(2) Departamento de Economía, Universidad de Jaén, Campus Las Lagunillas, Jaén, e-mail: jimenezf@ujaen.es

(3) Departamento de Economía Financiera y Contabilidad, Universidad de Jaén, Campus Las Lagunillas, Jaén, e-mail: mphorno@ujaen.es

(4) Departamento de Organización de Empresas, Marketing y Sociología, Universidad de Jaén, Campus Las Lagunillas, Jaén, e-mail: rmartine@ujaen.es

Presentado en eXIDO16 (2016)

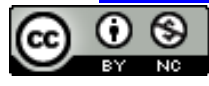

\section{RESUMEN}

El objetivo de este trabajo es cuantificar la percepción del alumnado de tercer curso de Grado sobre el nivel de adquisición de competencias genéricas alcanzadas hasta el momento. En concreto, son seis las competencias analizadas: capacidad de resolver problemas, capacidad de análisis y síntesis, capacidad de redactar informes, trabajo autónomo, comunicación oral y escrita y trabajo en equipo. Para ello, se han encuestado a 120 estudiantes de los Grados en Estadística y Empresa (EE) (14.17\%), Administración y Dirección de Empresas (ADE) (64.17\%) y Finanzas y Contabilidad (FC) $(21.67 \%)$. De los resultados obtenidos se desprende que existen diferencias significativas entre los tres Grados en relación a algunas de las competencias, como son: capacidad para resolver problemas (el $47 \%$ de los estudiantes de EE consideran que alcanzan un nivel alto en la aplicación del método apropiado para la resolución de un problema, frente al $23 \%$ en FC); capacidad de análisis y síntesis (el $76.47 \%$ de los encuestados de $\mathrm{EE}$ dicen tener un nivel alto para transmitir sintéticamente las ideas más relevantes, mientras que en $\mathrm{ADE}$ es el 29.87\%) y comunicación oral y escrita (el $70.6 \%$ de los estudiantes de EE se valoran con un nivel alto, siendo en FC un 42.3\%).

Palabras clave: competencias percibidas, planes de estudio universitarios.

\section{INTRODUCCIÓN}

Los actuales planes de estudios universitarios están orientados a la adquisición de las competencias y habilidades por parte de los estudiantes. De esta forma aunque no se excluye el tradicional enfoque basado en contenidos, lo que se hace en la actualidad es poner énfasis en los métodos de aprendizaje de dichas competencias. En la literatura se pueden consultar diversas definiciones del término competencia; no obstante, la mayoría de ellas se basan en la definición establecida en el Proyecto Tunning Educational Structures in Europe (González y Wagenaar, 2003) que se concreta como "una combinación dinámica de conocimiento, comprensión, habilidades y capacidades”.

Por otra parte, se espera que los graduados en títulos como Estadística y Empresa, Administración y Dirección de Empresas y Finanzas y Contabilidad, tengan que desarrollar su labor profesional en una empresa, organismo o institución 
dentro de un contexto multidisciplinar. Es por ello que deben de ser capaces de entender los distintos problemas planteados en diversos campos, realizar un análisis crítico y reflexión sobre la situación, así como elaborar informes y conclusiones que deben exponer y defender de manera adecuada tanto oral como de forma escrita. Todas estas competencias se recogen en las respectivas memorias de grado y se desarrollan dentro del contenido específico de las distintas asignaturas. Al mismo tiempo, al tratarse de competencias genéricas, pueden considerarse competencias transversales según la clasificación de Montero Curiel (2010: 28-29), comunes a la mayoría de las asignaturas y que se relacionan con la puesta en práctica integrada de conocimientos, aptitudes, rasgos de la personalidad y valores adquiridos. Es por ello que nos planteamos el objetivo de testar en el alumnado de tercer curso, cual es la percepción que tienen del nivel de adquisición de dichas competencias y las lagunas que encuentran en su formación que están relacionadas con la adquisición de las mismas.

Este trabajo se enmarca dentro de un proyecto de innovación docente que se desarrolla actualmente en la Universidad de Jaén, y que tiene como objetivo, facilitar el desempeño profesional de nuestros estudiantes, proponiendo actividades formativas complementarias en su último año de Grado, para que puedan reforzar y mejorar los aspectos deficitarios en cuanto a su formación y preparación al mundo laboral se refiere.

En las siguientes secciones se describe la metodología seguida, se comentan los principales resultados obtenidos y finalmente, se incluye un apartado con las conclusiones más relevantes.

\section{METODOLOGÍA}

Concretamente se ha medido la autoevaluación subjetiva de los estudiantes con respecto a seis competencias genéricas: capacidad de resolver problemas; capacidad de análisis y síntesis; capacidad de redactar informes; trabajo autónomo; comunicación oral y escrita y trabajo en equipo. Para ello, se ha diseñado un cuestionario de 22 ítems y se ha utilizado una escala de seis puntos: "nunca", "rara vez", "algunas veces", "bastantes veces", "casi siempre", y "siempre", respectivamente. El análisis en conjunto de los ítems asociados a una competencia, se ha realizado sumando las puntuaciones de los ítems implicados y atendiendo a su rango de posibles valores, se han dividido los resultados en tres categorías: "nivel bajo", "nivel aceptable" y "nivel alto" de adquisición, mediante la agrupación de dos en dos de las opciones iniciales.

La población objeto de estudio ha sido el tercer curso de los Grados en Estadística y Empresa (EE), Administración y Dirección de Empresas (ADE) y Finanzas y Contabilidad (FC). Se han encuestado a 120 estudiantes: el $14.17 \%$ de EE, el $64.17 \%$ de ADE y el $21.67 \%$ de FC.

\section{RESULTADOS}

Nuestros principales resultados ponen de manifiesto que efectivamente la mayoría de los alumnos encuestados son críticos con su percepción del nivel de adquisición de las competencias estudiadas. No obstante, hay ciertas peculiaridades que resaltar y que describimos a continuación.

Los porcentajes más altos de estudiantes encuestados que consideran haber adquirido un nivel alto de competencias corresponden a la "comunicación oral y escrita" con un $52.5 \%$ y al "trabajo en equipo" con un $42.5 \%$. Sin embargo, los porcentajes más bajos, para un nivel alto de competencias percibidas por parte de los estudiantes, son las correspondientes a “capacidad de análisis y síntesis" con un 19.17\% y "trabajo 
autónomo” con un $12.5 \%$.

Además, existen diferencias significativas entre los tres Grados en relación a algunas de las competencias. Es el caso de la capacidad para resolver problemas: el $47 \%$ de los estudiantes de EE consideran que alcanzan un nivel alto en la aplicación del método apropiado para la resolución de un problema, frente al 23\% en FC. También en la capacidad de análisis y síntesis se aprecian diferencias: el $76.47 \%$ de los encuestados de EE dicen tener un nivel alto para transmitir sintéticamente las ideas más relevantes, mientras que en $\mathrm{ADE}$ es el 29.87\%. En lo que se refiere a comunicación oral y escrita, el 70.6\% de los estudiantes de EE se valoran con un nivel alto, siendo en FC un 42.3\%. Es de destacar que en la capacidad para redactar informes, solo el $50 \%$ de los estudiantes de FC se valoran con nivel alto en el uso correcto de las normas gramaticales, el vocabulario adecuado y una redacción correcta.

\section{CONCLUSIONES}

Se ha cuantificado la percepción del alumnado de tercer curso de los Grados en EE, ADE y FC de la Universidad de Jaén, sobre el nivel de adquisición de competencias genéricas alcanzadas hasta el momento mediante un cuestionario. Las competencias analizadas han sido: capacidad de resolver problemas, capacidad de análisis y síntesis, capacidad de redactar informes, trabajo autónomo, comunicación oral y escrita y trabajo en equipo. Los resultados obtenidos sugieren que, en general, los alumnos encuestados son críticos con su percepción del nivel de adquisición de las competencias analizadas. Además, existen diferencias significativas entre los títulos de Grado considerados en relación a la valoración subjetiva de los estudiantes con respecto a las competencias: capacidad para resolver problemas, capacidad de análisis y síntesis y comunicación oral y escrita.

\section{REFERENCIAS}

González, J. y Wagenaar, R. (2003). Tuning Educational Structures in Europe. Universidad de Deusto. Disponible http://eua.be/eua/jsp/en/upload/TUNING_Announcement_Closing_Conference. 1084282515011.pdf.

Montero Curiel, M. (2010). El proceso de Bolonia y las nuevas competencias. Tejuelo: Didáctica de la Lengua y Literatura. Educación, 9, 19-37. 